\title{
ERRATUM
}

\section{Erratum to: Calculating entropies of alkaline earth metal molybdates}

\author{
Ernst Gamsjäger $^{1} \cdot$ Masao Morishita $^{2} \cdot$ Heinz Gamsjäger ${ }^{3}$
}

Published online: 25 May 2016

(C) Springer-Verlag Wien 2016

\section{Erratum to: Monatsh Chem (2016) 147:263-267 DOI 10.1007/s00706-015-1588-3}

The original version of this article unfortunately contained a mistake. The presentation of Eqs. 4, 5 and 9 was incorrect. These errata had no effect on the calculated heat capacities and entropies.

The correct versions are given below.

$$
\begin{aligned}
C_{p, \mathrm{~m}}^{\mathrm{o}}= & R\left[n_{\mathrm{D}} D\left(\theta_{\mathrm{D}} / T\right)+n_{\mathrm{E}} E\left(\theta_{\mathrm{E}} / T\right)+n_{\mathrm{S}} S\left(\theta_{\mathrm{S}} / T\right)\right] \\
C_{p, \mathrm{~m}}^{\mathrm{o}}= & R\left[n_{\mathrm{D}} D\left(\theta_{\mathrm{D}} / T\right)+n_{\mathrm{E}} E\left(\theta_{\mathrm{E}} / T\right)+X_{1} n_{\mathrm{S}} S\left(\theta_{\mathrm{S}} / T\right)\right] \\
& +\left(1-X_{1}\right) \cdot\left(A_{1} T+A_{2} T^{2}\right)+X_{2} \cdot\left(B_{1} T+B_{2} T^{2}\right) \\
S_{T}^{\mathrm{o}} / R= & \int_{0}^{T_{\text {ref }}}\left\{\begin{array}{l}
{\left[n_{\mathrm{D}} D\left(\theta_{\mathrm{D}} / T\right)+n_{\mathrm{E}} E\left(\theta_{\mathrm{E}} / T\right)+X_{1} n_{\mathrm{S}} S\left(\theta_{\mathrm{S}} / T\right)\right] / T} \\
+\left[\left(1-X_{1}\right) \cdot\left(A_{1}+A_{2} T\right)+X_{2} \cdot\left(B_{1}+B_{2} T\right)\right] / R
\end{array}\right\} \mathrm{d} T
\end{aligned}
$$

The online version of the original article can be found under doi:10.1007/s00706-015-1588-3.

\section{Heinz Gamsjäger}

gamsjaeg@unileoben.ac.at

1 Institut für Mechanik, Montanuniversität Leoben, 8700

Leoben, Austria

2 Department of Materials Science and Chemistry, University of Hyogo, Hyogo 671-2280, Japan

3 Lehrstuhl für Physikalische Chemie, Montanuniversität, 8700 Leoben, Austria 